\title{
Magnitude of Antiretroviral Drug Toxicity in Adult HIV Patients in Ethiopia: A cohort study at seven teaching hospitals
}

\author{
Esayas Kebede Gudina ${ }^{1^{*}}$, Alula M. Teklu ${ }^{2}$, Asres Berhan ${ }^{3}$, Atsbeha \\ Gebreegziabhier $^{4}$, Teshome Seyoum ${ }^{5}$, Abiy Nega ${ }^{2}$, Girmay Medhin ${ }^{6}$, Amha \\ Kebede $^{4}$, Yibeltal Assefa ${ }^{4}$
}

\footnotetext{
OPEN ACCESS

Citation: Esayas Kebede Gudina, Alula M. Teklu, Asres Berhan, et al. Magnitude of Antiretroviral Drug Toxicity in Adult HIV Patients in Ethiopian: A cohort study at seven teaching hospitals. Ethiop J Health Sci 2017;27(si1):39-52. doi: http://dx.doi.org/10.4314/ejhs.v27i1.5S.

Received: February 1, 2016

Accepted: August 12, 2016

Published: March 15, 2017

Copyright: ( 2017 Esayas KG et al. This is an open access article distributed under the terms of the Creative Commons Attribution License, which permits unrestricted use, distribution, and reproduction in any medium, provided the original author and source are credited.

Funding:Center for Disease Control and Prevention (CDC)

Competing Interests: The authors

declare that this manuscript was approved by all authors in its form and that no competing interest exists.

Affilation and Correspondence:

${ }^{1}$ Department of Internal Medicine, Jimma University, Jimma

${ }^{2}$ MERQ Consultancy Services, Addis Ababa, Ethiopa

${ }^{3}$ Department of Pharmacology, Hawassa University, Hawassa

${ }^{4}$ Ethiopian Public Health Institute, Addis Ababa

${ }^{5}$ Department of Internal Medicine, Hiwot Fana Hospital, Harar

${ }^{6}$ Aklilu Lemma Institute of

Pathobiology, Addis Ababa

"Esayas Kebede Gudina, E-mail: esakgd@gmail.com
}

\begin{abstract}
Background: The introduction of antiretroviral therapy (ART) has resulted in significant mortality reduction and improvement in the quality of life. However, this has come at a cost of increased drug toxicity. The objective of this study was to assess the patterns and predictors of ART toxicity in adult HIV patients in Ethiopia.

Methods: This is a prospective cohort study conducted at seven teaching hospitals between September 2009 and December 2013 involving $3921 \mathrm{HIV}$ patients on ART. Adverse drug reactions (ADR) due to ART were identified based on clinical assessment and/or laboratory parameters. Multivariable random effects Poisson regression analysis was used to identify factors independently associated with toxicity.

Result: ADR due to ART drugs was reported in 867 (22.1\%) of the participants; 374 (9.5\%) had severe forms. About $87 \%$ of reported toxicities were limited to three organ systems - the skin, nervous system and blood. The overall incidence of ADR was 9 per 100 person years. About a third of toxicities occurred during the first six months after ART initiation with the incidence rate of 22.4 per 100 person years. Concomitant anti-tuberculosis treatment was the strongest independent predictor of toxicity.

Conclusion: $A D R$ was found to be highly prevalent in HIV patients on ART at tertiary hospitals in Ethiopia. Most of these conditions occurred early after ART initiation and in those with concomitant anti-tuberculosis treatment. Thus, routine monitoring of patients on ART should be strengthened with particular emphasis in the first 6 months. Strategies should also be devised to replace older and more toxic agents with newer and safer drugs available.

Key words: HIV, ART, adverse drug reaction, incidence rate, ACM, Ethiopia
\end{abstract}




\section{INTRODUCTION}

Human immunodeficiency Virus (HIV) has caused significant socioeconomic crisis for the last few decades in sub-Saharan Africa (1). The discovery of antiretroviral therapy (ART) was the breakthrough in care and treatment of people living with HIV, resulting in reduction in mortality and improvement in the quality of life. This has transformed HIV from untreatable condition into a chronic manageable disease (2). However use of ART and other drugs used in HIV care has resulted in an increment in serious adverse drug reactions (ADR) some of which are lifethreatening conditions $(3,4)$.

Adverse drug reaction is a response to a drug which is noxious and unintended. It is drug toxicity which occurs at doses normally used for prophylaxis, diagnosis, or treatment (5). Among HIV patients, the possible causes for drug toxicity are antiretroviral (ARV) drugs themselves; drug used for prophylaxis and treatment of opportunistic infections (OI) and other concomitant medications $(6,7)$. Adverse reactions occur with all ARV drugs and are among the major reasons for switching or discontinuing therapy as well as for medication non-adherence (8-10). ADRs have thus become one of the most important limiting factors to the successful treatment of HIV (11-13).

The magnitude of the problem significantly differs between developed and developing world due to various reasons (7). Toxicities related to ART in the developing world, in sub-Saharan Africa in particular, are more prevalent because the regimens contain older and more toxic agents like stavudin (d4t), zidovudin (AZT) and nevirapin (NVP). In addition to these, patients late presentation with advanced disease state, occurrence of opportunistic infections and comorbid medical conditions like malnutrition have made the problem even worse $(6,7,14)$. Lack of laboratory monitoring and skilled professionals may also result in failure or delay in diagnosing specific toxicities and hence increasing their severity $(6,7,12)$.

In the year 2013, about 790,000 people were estimated to live with HIV in Ethiopia; with adult prevalence of $1.2 \%$ and 45,000 deaths annually (15). Routine HIV care and free antiretroviral therapy (ART) services started in the country in 2005 (16). This has resulted in a marked decrement in morbidities and mortalities related to the disease and improvement in quality of life. There are currently about 320,000 adults receiving ART in Ethiopia (17). Few studies in the country have shown that ARV drug toxicity in Ethiopia is a common occurrence and major reason for ART regiment change or modification $(9,18,19)$. However, most of the studies lack generalizability due to small sample size focusing on limited facility in central Ethiopia. The aim of this study was thus to assess the magnitude and patterns of ARV drug toxicity in adult HIV patients at major tertiary hospitals in Ethiopia.

\section{METHODS}

Study design and setting: This study is part of Advanced Clinical Monitoring (ACM) multicenter cohort. The ACM cohort was conducted in Ethiopia at seven teaching Hospitals located at various parts of the country (Addis Ababa, Hawassa, Jimma, Haramaya, Mekelle, Gondar and Army forces hospital). The cohort included both ART naïve and ART experienced adult and pediatric HIV positive patients.

This article presents findings of adverse drug reaction in adult HIV patients on ART. To involve all patients who were started on ART since the beginning of HIV care in Ethiopia, both retrospective and prospective cohort study designs were used. In the retrospective design, data was obtained from medical records of ART experienced patients since initiation of ART which covers the period of January 2005 to August 2009. The other group consisted of ART naive patients who were enrolled since 2009. ART experienced patients who were still alive and on follow-up at the beginning of the prospective design were followed in the same way as prospective group.

Baseline data collected from participants included socio-demographic profile, general medical conditions, laboratory parameters and components of HIV care given to them (ART regimen, treatment for OI and prophylaxis given). Participants were subsequently followed for occurrence of adverse reactions related to ART

DOI: http://dx.doi.org/10.4314/ejhs.v27i1.5S 
using clinical parameters and laboratory data where applicable. A particular emphasis was given to the first 6 months after ART initiation as most of the toxicities tend to occur during this time (7). These data were obtained from medical records for patients who were started on ART before 2009 (before recruitment started).

Assessment for ADR was done by physician working at ART clinics at respective hospitals. Patients were assessed on each visit using checklists prepared for routine follow-up evaluation. National guidelines (and WHO recommendations if applicable) for timing of hematologic and blood chemistry evaluation were followed. In patients with suspected ADR, all necessary laboratory tests were done as needed.

Inclusion and Exclusion Criteria: To assess ART related adverse drug reactions, this study included all adult ACM cohort participants who were either ART naïve or ART experienced HIV positive patients at the start of the cohort. Only participants of age 18 years or older at enrollment were included in this study. Accordingly, a total of 3921 adult HIV positive patients from seven teaching hospitals in Ethiopia were included in this study for analysis of ART drug toxicity. Large proportion of the participants, 2304 (58.8\%) belonged to ART experienced group.

Patients who died or were transferred out within a week of ART initiation and those who were on ART for the same duration at the last recruitment were not included in this study.

Definition of outcome variables: The outcome of interest in this study was drug toxicities. An algorithm was developed to identify drug toxicities meeting the operational definition based on World Health Organization (WHO) criteria for ADR (5) (Annex 1).

Adverse drug reactions in patients on ART were identified based on clinical and/or laboratory data. Only clinical conditions known to arise from potential agent, in the absence of other compelling medical conditions, were attributable to ADR. Mild medical conditions which commonly occur in the general public but can be attributable to the drugs were excluded from this list unless specified and there was clear evidence for direct contribution by the agent. In most cases this refers to gastrointestinal disturbances (dyspeptic symptoms) which in most of the patients have been reported before or after ART initiation. Major gastrointestinal upset reported by patient to be worsened after these medication or has resulted in their discontinuation due to these conditions were considered as evident drug toxicity.

Severe or life threatening toxicity was defined based on clinical or laboratory evidences classified as grade 3 or 4 by WHO criteria (Annex - 1). All forms of toxicities resulting in hospitalization, major disability, changing or discontinuation of the culprit agent were categorized in this group.

In cases of multiple occurrences of toxicities in a single patient, the count was based on the systems involved and time of occurrence. Multisystem complications that were due to single mechanism were counted as one (e.g lactic acidosis and pancreatitis due to stavudin were considered as related toxicities if occurred within one week of each other). However, if the pathophysiologic mechanisms were different, they were counted separately (e.g., anemia and lactic acidosis in zidovudin were considered as separate forms of toxicities). Similar toxicities, toxicities of similar pathophysiology or of the same spectrum occurring within one week of each other and nonresolving ones were counted as one type of toxicity.

All forms of toxicity were classified based on their severity and body system affected.

Data quality assurance: Data collectors were trained and regularly supervised by Johns Hopkins Technical Support for the Ethiopian HIV/AIDS Initiative (TSEHAI). Refresher trainings were given to sites based on gaps identified. Standard reporting formats were used to minimize interpersonal variation and Variation between sites. All incoming staffs (physicians and nurse) were trained on entry.

Data management and Data analysis: The data was initially computerized using Microsoft access. This data was exported to SPSS version 20 and STATA version 12 for analysis. Tableau version 8 was also used to portray the trends in toxicity.

Because toxicities are more likely to occur during the initial period of exposure to ART, we calculated the six-month incidence of drug toxicity as a probability of a participant experiencing one or more drug toxicities during the first six months from initiation of ART. The crude incidence rates

DOI: http://dx.doi.org/10.4314/ejhs.v27i1.5S 
of toxicity per 100 person-years on ART was calculated by summing the total number of occurrences of toxicity and dividing this total by the summed person-time of each participant from ART start to either the time patient left the study or study terminated.

Incidence of toxicity was estimated using random effects multivariable Poisson regression analysis. Potential risk factors to be included in multivariable model were selected if $p$-value in the bivariate analysis was less than $20 \%$. Clustering at site level was adjusted both in univariate and multivariable analysis.

We also calculated stratified incidence rates for each year that patients took ART. It was acknowledge that each participant might have one or more incidence of toxicity. The six-month incidence and incidence rates of all toxicities combined and of each severity category was calculated separately. Homogeneity of the 6month incidence across sites was assessed using the Breslow-Day test and homogeneity of the incidence rates across sites was assessed using a chi-square test.

Ethical considerations: Ethical clearance of the study was obtained from Ethiopian Public Health
Institute, Minister of Science and Technology of Ethiopia and Center for Diseases Control and prevention (CDC), Atlanta, USA. Written informed consent was obtained from all participants before recruitment. Data was collected using numerical codes and will remain anonymous.

\section{RESULTS}

Baseline characteristics: ACM cohort of 3921 adult HIV patients who were on ART were assessed for the occurrence of ART drug toxicity; $62 \%$ of them were women. The mean age at ART initiation was 34.5 years $(\mathrm{SD}=9.2)$ and $75 \%$ were between 25 and 44 years of age. More than 60\% of the participants had baseline body weight of less than 55 kilograms. Nearly $60 \%$ of the participants reported use of some form of substance; $6.7 \%$ have used hard drugs including morphine and cocaine. The majority of the participants were at advanced staged of their HIV at initiation of ART; $65.9 \%$ had WHO stage $\geq 3$ disease and $69.7 \%$ had CD4 count of $<200$. In terms of functional status, $4.4 \%$ of the patients were bedridden at baseline (Table 1).

Table 1: Baseline characteristics of adult HIV patients at initiation of ART in HIV patients at tertiary hospitals in Ethiopia.

\begin{tabular}{crrrrrr}
\hline & \multicolumn{2}{c}{ Total participant } & \multicolumn{4}{c}{ Toxicity } \\
\cline { 2 - 7 } Age category (N=3921) & & & Yes & No \\
\cline { 2 - 7 } & Number & \% & Number & \% & Number & \% \\
\hline$<25$ & 399 & 10.2 & 92 & 23.1 & 307 & 76.9 \\
$25-34$ & 1761 & 44.9 & 386 & 21.9 & 1375 & 78.1 \\
$35-44$ & 1181 & 30.1 & 248 & 21.0 & 933 & 79.0 \\
$>=45$ & 580 & 14.8 & 141 & 24.3 & 439 & 75.7 \\
\hline Gender (N=3921) & & & & & & \\
Female & 2426 & 61.9 & 609 & 25.1 & 1817 & 74.9 \\
Male & 1495 & 38.1 & 258 & 17.3 & 1237 & 82.7 \\
\hline Baseline body weight, Kg (N=3738) & & & & & & \\
<45 & 802 & 20.5 & 209 & 26.1 & 593 & 73.9 \\
45-54 & 1547 & 39.5 & 359 & 23.2 & 1188 & 76.8 \\
55-64 & 970 & 24.7 & 197 & 20.3 & 773 & 79.7 \\
>65 & 419 & 10.7 & 69 & 16.5 & 2350 & 83.5 \\
\hline ART history (N=3921) & & & & & & \\
ART experienced & 2304 & 58.8 & 642 & 27.9 & 1662 & 72.1 \\
ART naïve & 1617 & 41.2 & 225 & 13.9 & 1392 & 86.1 \\
\hline
\end{tabular}

DOI: http://dx.doi.org/10.4314/ejhs.v27i1.5S 
Table 1. continued...

\begin{tabular}{|c|c|c|c|c|c|c|}
\hline \multicolumn{7}{|l|}{ Educational status ( $\mathrm{N}=3843$ ) } \\
\hline No education & 723 & 18.4 & 189 & 26.1 & 534 & 73.9 \\
\hline Primary & 1393 & 35.5 & 304 & 21.8 & 1089 & 78.2 \\
\hline Secondary & 1310 & 33.4 & 276 & 21.1 & 1034 & 78.9 \\
\hline Tertiary & 417 & 10.6 & 78 & 18.7 & 339 & 81.3 \\
\hline \multicolumn{7}{|l|}{ Employment status $(\mathrm{N}=\mathbf{3 2 8 5})$} \\
\hline Unemployed & 1571 & 40.1 & 374 & 23.8 & 1197 & 76.2 \\
\hline Too sick to work & 385 & 9.8 & 92 & 23.9 & 293 & 76.1 \\
\hline Working part-time & 91 & 2.3 & 20 & 22.0 & 71 & 78 \\
\hline Working full time & 1225 & 31.2 & 230 & 18.8 & 995 & 81.2 \\
\hline Other & 13 & 0.3 & 3 & 23.1 & 10 & 76.9 \\
\hline \multicolumn{7}{|l|}{ Marital status $(\mathrm{N}=\mathbf{3 8 7 0})$} \\
\hline Never married & 663 & 16.9 & 122 & 18.4 & 541 & 81.6 \\
\hline Married(Inc. de facto) & 1834 & 46.8 & 358 & 19.5 & 1476 & 80.5 \\
\hline Separated or Divorced & 803 & 20.5 & 218 & 27.1 & 585 & 72.9 \\
\hline Widow/Widower & 570 & 14.5 & 151 & 26.5 & 419 & 73.5 \\
\hline \multicolumn{7}{|l|}{ Facility (Hospital) $(\mathrm{N}=3921)$} \\
\hline Tikur Anbessa & 566 & 14.4 & 165 & 29.2 & 401 & 70.8 \\
\hline Army & 557 & 14.2 & 53 & 9.5 & 504 & 90.5 \\
\hline Gondar & 610 & 15.6 & 155 & 25.4 & 455 & 74.6 \\
\hline Jimma & 540 & 13.8 & 83 & 15.4 & 457 & 84.6 \\
\hline Mekelle & 582 & 14.8 & 178 & 30.6 & 404 & 69.4 \\
\hline Haramaya & 530 & 13.5 & 129 & 24.3 & 401 & 75.7 \\
\hline Hawassa & 536 & 13.7 & 104 & 19.4 & 432 & 80.6 \\
\hline \multicolumn{7}{|l|}{ WHO staging $(\mathrm{N}=3852)$} \\
\hline 1 and 2 & 1265 & 32.8 & 253 & 20.0 & 1012 & 80.0 \\
\hline 3 and 4 & 2587 & 67.2 & 598 & 23.1 & 1989 & 76.9 \\
\hline \multicolumn{7}{|l|}{ CD4 count category $(\mathrm{N}=3789)$} \\
\hline$<200$ & 2735 & 72.2 & 581 & 21.2 & 2154 & 78.8 \\
\hline 200-349 & 983 & 25.9 & 232 & 23.6 & 751 & 76.4 \\
\hline$\geq 350$ & 71 & 1.9 & 17 & 23.9 & 54 & 76.1 \\
\hline \multicolumn{7}{|l|}{ Functional status (N=3840) } \\
\hline Working & 2796 & 71.3 & 631 & 22.6 & 2165 & 77.4 \\
\hline Ambulatory & 872 & 22.2 & 199 & 22.8 & 673 & 77.2 \\
\hline Bedridden & 172 & 4.4 & 23 & 13.4 & 149 & 86.6 \\
\hline \multicolumn{7}{|c|}{ ART regiment at initiation ( $\mathrm{N}=3918$ ) } \\
\hline $\mathrm{AZT}+3 \mathrm{TC}+\mathrm{EFV}$ & 434 & 11.1 & 87 & 20.0 & 347 & 80.0 \\
\hline $\mathrm{AZT}+3 \mathrm{TC}+\mathrm{NVP}$ & 1053 & 26.9 & 237 & 22.5 & 816 & 77.5 \\
\hline $\mathrm{D} 4 \mathrm{~T}+3 \mathrm{TC}+\mathrm{EFV}$ & 344 & 8.8 & 93 & 27.0 & 251 & 73.0 \\
\hline $\mathrm{D} 4 \mathrm{~T}+3 \mathrm{TC}+\mathrm{NVP}$ & 951 & 24.3 & 289 & 30.4 & 662 & 69.6 \\
\hline $\mathrm{TDF}+3 \mathrm{TC}+\mathrm{EFV}$ & 920 & 23.5 & 122 & 13.3 & 798 & 86.7 \\
\hline $\mathrm{TDF}+3 \mathrm{TC}+\mathrm{NVP}$ & 209 & 5.3 & 39 & 18.7 & 170 & 81.3 \\
\hline Others & 7 & 0.18 & 0 & 0 & 7 & 100.0 \\
\hline
\end{tabular}

The initial ART regimen in all patients was a combination of two nucleoside reverse transcriptase inhibitors (NRTI) and one nonnucleoside reverse transcriptase inhibitor (NNRTI). The most commonly prescribed NRTIs were AZT (38\%) and d4t (33.1\%). However, this trend changed after the introduction of tenofovir (TDF) in 2009 which was used as an initial NRTI in $53.6 \%$ of the cases since then. Efavirinez (EFV) and NVP were the only NNRTs prescribed; the

DOI: http://dx.doi.org/10.4314/ejhs.v27i1.5S 
latter was used in $56.5 \%$ of the patients. All but one patient took lamivudine (3TC) as the backbone of the entire regimen.

Over $90 \%$ (3585) of them were on cotrimoxazole prophylactic treatment (CPT) while only $4.1 \%$ (160) were prescribed isoniazid (INH) preventive therapy (IPT) at time of ART initiation; $15.4 \%$ (604) were taking anti-tuberculosis treatment.

The mean follow-up period of patients on ART was 43.7 ( $\mathrm{SD}=28.7$ ) months with median follow-up of 41.6 months. There was significant difference in the mean follow-up of patients in Experienced group $($ mean $=60.3 ; \mathrm{SD}=24.5$; median $=64.8$ months $)$ and Naïve group $($ mean $=$ 19.2; $\mathrm{SD}=13.1$; median 18.5 months).

Patterns of adverse drug reaction: Overall, there were 1253 incidents of ADR reported during the follow-up period. Of these, $542(43.3 \%)$ were severe or life threatening toxicities. The rate of toxicity was the highest in the first 6 months after ART initiation; $31.8 \%$ occurred during this period with the trend gradually decreasing overtime (Fig 1). In general, ADR was reported in 867 (22.1\%) of the participants over the follow-up period; 374 $(9.5 \%)$ had severe forms. Of those with ADR, 240 $(27.7 \%)$ had more than one incident.

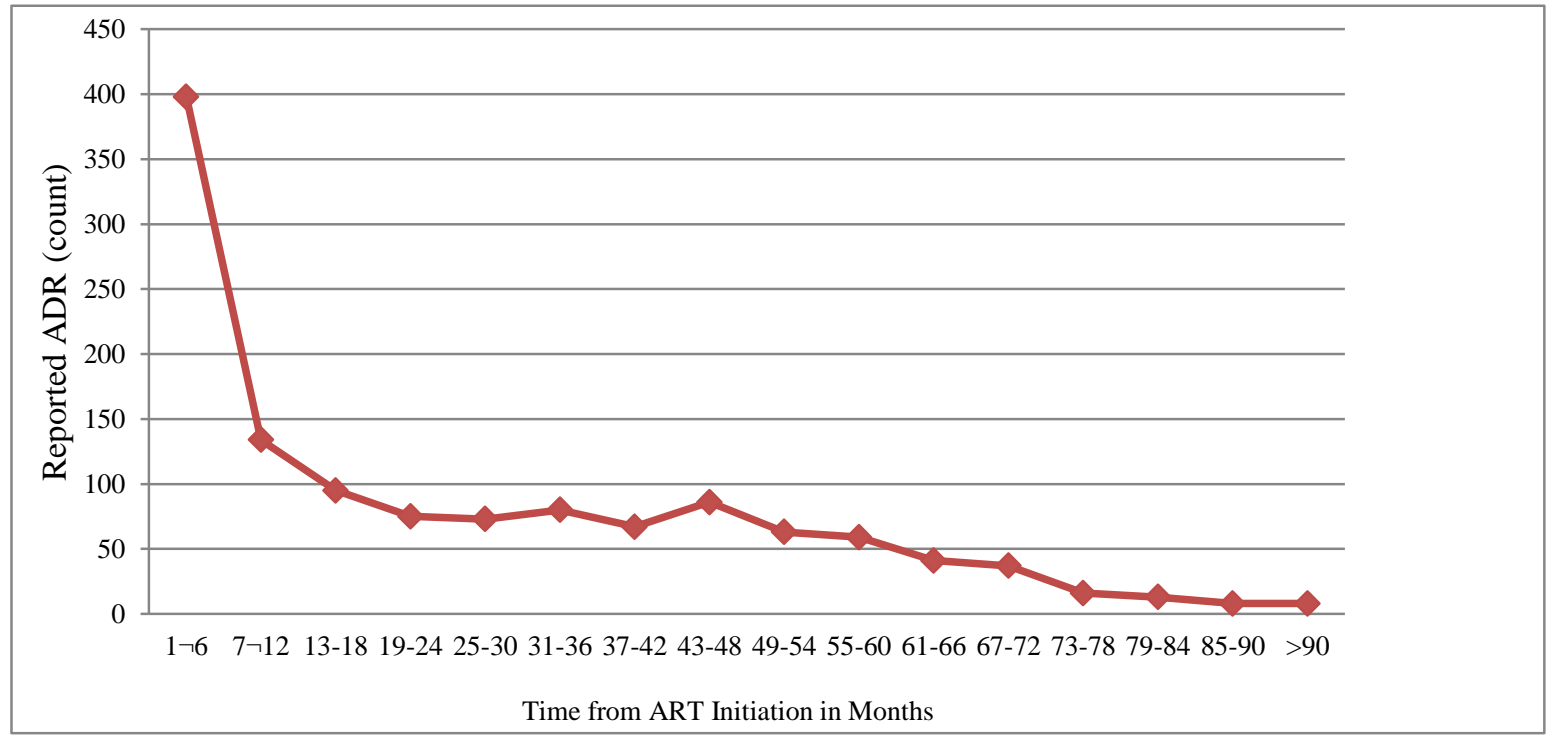

Figure 1: Trends in occurrence of ADR in adult HIV patients on ART at tertiary hospitals in Ethiopia

The incidence rate of ADR over follow-up time was 9 per 100 person years. The incidence of toxicities was highest in the first 6 months of ART initiation with incidence rate of 22.4 per 100 person years.

About $87 \%$ of reported toxicities were limited to three organ systems - the skin $(36.6 \%)$, nervous system $(35.4 \%)$ and blood (14.9\%). While most of dermatologic ADR were milder forms, about
$67.0 \%$ of severe forms of adverse events were neurologic (Fig 2). Moreover, over $80 \%$ of neurologic toxicities were classified as severe forms which have resulted in either disabilities or hospitalization or regimen change. Most of the patients with neurologic toxicities and all with severe forms were taking $\mathrm{d} 4 \mathrm{t}$ at the time of the toxicity incidence. 


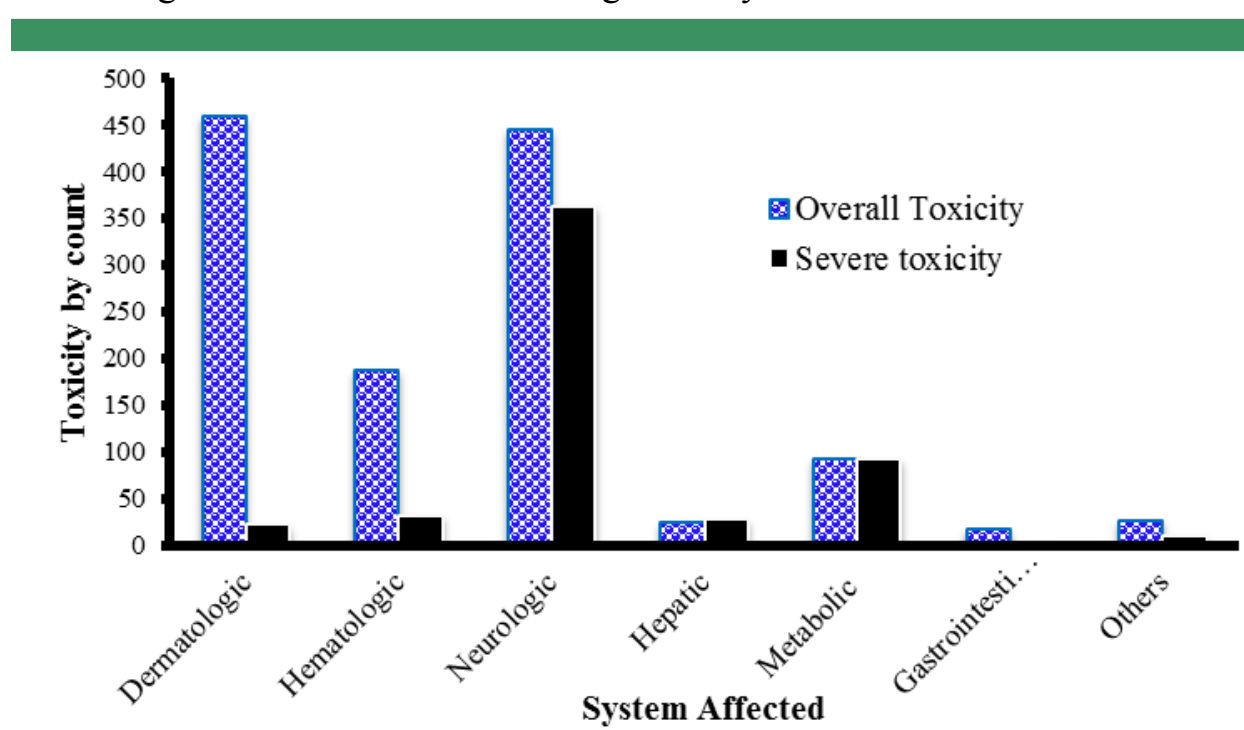

Figure 2: Types of ADR by system affected in HIV patients on ART at tertiary hospitals in Ethiopia

The incidence rate of adverse events in the first six months by year of ART start $(2005$ - 2013) ranged from 11.9 to 36.8 per 100 person year. In general, the 6 months and overall incidence rate of ADR were higher in patients who started on ART since the 2009 as compared those who started earlier (Table 2).

Table 2: Incident Rate of Toxicity by Year of ART Start at six month and over all follow-up period in HIV patients at tertiary hospitals in Ethiopia.

\begin{tabular}{lrrrrrr}
\hline & \multicolumn{3}{c}{ Within 6 month } & \multicolumn{3}{c}{ Overall } \\
\cline { 2 - 7 } $\begin{array}{l}\text { ART } \\
\text { start } \\
\text { year }\end{array}$ & $\begin{array}{l}\text { Follow Up } \\
\text { Time (in } \\
\text { days }\end{array}$ & $\begin{array}{l}\text { No. of } \\
\text { Toxicity }\end{array}$ & $\begin{array}{l}\text { Incident rate } \\
\text { (Per 100 } \\
\text { Person-Year) }\end{array}$ & $\begin{array}{l}\text { Follow } \\
\text { Up Time }\end{array}$ & $\begin{array}{l}\text { No. of } \\
\text { Toxicity }\end{array}$ & $\begin{array}{l}\text { Incident rate } \\
\text { (Per 100 Person- } \\
\text { Year) }\end{array}$ \\
\hline 2005 & 41378 & 21 & 18.5 & 586067 & 103 & 6.4 \\
2006 & 110812 & 36 & 11.9 & 1380500 & 277 & 7.3 \\
2007 & 92257 & 52 & 20.6 & 993457 & 256 & 9.4 \\
2008 & 79201 & 49 & 22.6 & 718597 & 174 & 8.8 \\
2009 & 85133 & 58 & 24.9 & 584458 & 168 & 10.5 \\
2010 & 114886 & 88 & 28.0 & 555865 & 152 & 10.0 \\
2011 & 58475 & 59 & 36.8 & 193012 & 84 & 15.9 \\
2012 & 52531 & 29 & 20.2 & 101332 & 33 & 11.9 \\
2013 & 14670 & 6 & 14.9 & 15669 & 6 & 14.0 \\
\hline
\end{tabular}

Predictors of adverse drug reaction: Multivariable random effect Poisson regression analysis was done to find predictors for occurrences of ADR in the first six months of HAART initiation. Patients who started ART in 2009 and after had higher rates of ADR as compared to those who started treatment prior to that; AIRR 1.38, 95\% CI $1.017-1.87$ and 1.49 ,
95\% CI 1.03 - 2.15 for 2009-2010 and 2011-2013 respectively as compared to ART start year of 2005-2008. Similarly, lower literacy status, marital inconvenience (being divorced or separated), and concomitant anti-TB treatment were independent predictors of ADR in the first 6 months (Table 3). 
Similar analysis for patients started on HAART since 2009 (prospectively recruited patients) showed that lower educational status, lower CD4

count $(<200)$, and concomitant TB treatment were independent predictors of ADR (Table 4).

Table 3: Predictors of Toxicity within 6 months of ART initiation in HIV patients at tertiary hospitals in Ethiopia: both ART naïve and experienced groups.

\begin{tabular}{|c|c|c|c|c|c|}
\hline \multirow[t]{2}{*}{ Characteristic } & & \multicolumn{2}{|c|}{ Uni-variable analysis } & \multicolumn{2}{|c|}{ Multivariable analysis } \\
\hline & & IRR $(95 \%$ CI $)$ & P-value & IRR $(95 \%$ CI $)$ & P -Value \\
\hline \multirow[t]{2}{*}{ Sex } & Female & 1 & & 1 & \\
\hline & Male & $0.803(0.640-1.007)$ & 0.057 & $0.825(0.630-1.079)$ & 0.160 \\
\hline \multirow{4}{*}{$\begin{array}{l}\text { Age at ART } \\
\text { start }\end{array}$} & $18-24$ & 1 & & & \\
\hline & $25-34$ & $1.159(0.795-1.688)$ & 0.443 & & \\
\hline & $35-44$ & $1.047(0.703-1.559)$ & 0.823 & NS & \\
\hline & $45+$ & $1.245(0.809-1.915)$ & 0.319 & & \\
\hline \multirow{3}{*}{$\begin{array}{l}\text { ART Start } \\
\text { Year }\end{array}$} & $2005-2008$ & 1 & & 1 & \\
\hline & 2009-2010 & $1.524(1.202-1.932)$ & 0.000 & $1.378(1.017-1.868)$ & $0.039 *$ \\
\hline & 2011-2013 & $1.533(1.173-2.003)$ & 0.002 & $1.487(1.029-2.151)$ & $0.035^{*}$ \\
\hline \multirow{4}{*}{$\begin{array}{l}\text { Educational } \\
\text { status }\end{array}$} & No education & 1 & & 1 & \\
\hline & Primary & $0.724(0.545-0.961)$ & 0.025 & $0.729(0.543-0.980)$ & $0.036^{*}$ \\
\hline & Secondary & $0.867(0.656-1.145)$ & 0.314 & $0.851(0.633-1.144)$ & 0.284 \\
\hline & Tertiary & $0.807(0.545-1.194)$ & 0.283 & $0.866(0.573-1.309)$ & 0.495 \\
\hline \multirow[t]{4}{*}{ Marital Status } & Never married & 1 & & 1 & \\
\hline & Married(Inc. de facto) & $1.145(0.827-1.585)$ & 0.416 & $1.093(0.778-1.537)$ & 0.608 \\
\hline & Separated or Divorced & $1.663(1.181-2.340)$ & 0.004 & $1.545(1.070-2.230)$ & $0.020 *$ \\
\hline & Widow/Widower & $1.027(0.687-1.534)$ & 0.898 & $1.100(0.719-1.683)$ & 0.659 \\
\hline Weight at & $<=45$ & 1 & & 1 & \\
\hline \multirow[t]{4}{*}{ ART Start } & $46-51$ & $0.973(0.726-1.304)$ & 0.855 & $1.075(0.791-1.460)$ & 0.645 \\
\hline & $52-58$ & $0.935(0.693-1.263)$ & 0.663 & $1.011(0.732-1.397)$ & 0.947 \\
\hline & $59+$ & $1.224(0.905-1.655)$ & 0.190 & $1.323(0.936-1.869)$ & 0.113 \\
\hline & $\mathrm{I}$ & 1 & & & \\
\hline Base WHO & II & $1.291(0.870-1.915)$ & 0.205 & & \\
\hline \multirow[t]{2}{*}{ Stage } & III & $1.034(0.721-1.482)$ & 0.858 & NS & \\
\hline & IV & $1.107(0.723-1.694)$ & 0.640 & & \\
\hline Base & Working & 1 & & 1 & \\
\hline Functional & Ambulatory & $0.935(0.715-1.221)$ & 0.621 & $1.019(0.759-1.370)$ & 0.899 \\
\hline Status & Bedridden & $0.535(0.254-1.128)$ & 0.100 & $0.539(0.221-1.314)$ & 0.174 \\
\hline Base CD4 & $<=50$ & 1 & & & \\
\hline \multirow[t]{4}{*}{ Count } & $51-100$ & $0.958(0.659-1.393)$ & 0.823 & & \\
\hline & $101-200$ & $0.905(0.646-1.266)$ & 0.559 & NS & \\
\hline & $201-350$ & $1.240(0.879-1.750)$ & 0.220 & & \\
\hline & $350+$ & $1.433(0.674-3.049)$ & 0.350 & & \\
\hline Base & TDF-NVP & 1 & & 1 & \\
\hline \multirow[t]{5}{*}{ Regimen } & TDF-EFV & $0.745(0.500-1.111)$ & 0.149 & $0.650(0.428-0.986)$ & $0.043 *$ \\
\hline & ZDV-EFV & $0.649(0.418-1.008)$ & 0.054 & $0.687(0.426-1.107)$ & 0.123 \\
\hline & ZDV-NVP & $0.559(0.380-0.821)$ & 0.003 & $0.596(0.396-0.897)$ & $0.013 *$ \\
\hline & d4T-EFV & $0.443(0.260-0.756)$ & 0.003 & $0.551(0.304-0.997)$ & $0.049 *$ \\
\hline & d4T-NVP & $0.487(0.323-0.736)$ & 0.001 & $0.573(0.351-0.935)$ & $0.026 *$ \\
\hline \multirow[t]{2}{*}{$\mathrm{CPT}$} & Yes & $0.864(0.602-1.239)$ & 0.426 & $\mathrm{NS}$ & \\
\hline & No & 1 & & & \\
\hline \multirow[t]{2}{*}{ IPT } & Yes & $0.853(0.466-1.561)$ & 0.606 & NS & \\
\hline & No & 1 & & & \\
\hline \multirow[t]{2}{*}{ TB Treatment } & Yes & $1.439(1.124-1.843)$ & 0.004 & $1.443(1.081-1.926)$ & $0.013 *$ \\
\hline & No & 1 & & 1 & \\
\hline
\end{tabular}

DOI: http://dx.doi.org/10.4314/ejhs.v27i1.5S 
When severe forms of toxicity were analyzed separately, marital inconvenience (being divorced or separated) and concomitant $\mathrm{TB}$ treatment remained independent predictors. AZT-EVF containing regimen was also found to result in $71 \%$ decrement in severe toxicity as compared to TDF-NVP containing regimen (Table 5).

Table 4: Predictors of Toxicity within 6 months of ART initiation in HIV patients at tertiary hospitals in Ethiopia: ART naïve group.

\begin{tabular}{|c|c|c|c|c|c|}
\hline \multirow[t]{2}{*}{ Characteristics } & & \multicolumn{2}{|c|}{ Uni-variable analysis } & \multicolumn{2}{|c|}{ Multivariable analysis } \\
\hline & & IRR $(95 \%$ CI $)$ & P-Value & IRR $(95 \%$ CI $)$ & P-Value \\
\hline \multirow[t]{2}{*}{ Sex } & Female & 1 & & 1 & \\
\hline & Male & $0.63(0.446,0.901)$ & 0.011 & $0.71(0.465,1.077)$ & 0.107 \\
\hline \multirow[t]{4}{*}{ Age ART Start } & $18-24$ & 1 & & & \\
\hline & $25-34$ & $1.38(0.744,2.577)$ & 0.304 & & \\
\hline & $35-44$ & $1.08(0.560,2.063)$ & 0.828 & & \\
\hline & $45+$ & $1.22(0.601,2.492)$ & 0.579 & & \\
\hline \multirow[t]{5}{*}{ ART Start Year } & 2013 & 1 & & 1 & \\
\hline & 2009 & $1.60(0.548,4.680)$ & 0.389 & $1.20(0.351,4.126)$ & 0.768 \\
\hline & 2010 & $1.66(0.719,3.819)$ & 0.235 & $1.51(0.593,3.849)$ & 0.387 \\
\hline & 2011 & $2.12(0.907,4.973)$ & 0.083 & $2.15(0.841,5.511)$ & 0.110 \\
\hline & 2012 & $1.30(0.537,3.136)$ & 0.563 & $0.96(0.356,2.591)$ & 0.937 \\
\hline \multirow[t]{4}{*}{ Education } & No education & 1 & & 1 & \\
\hline & Primary & $0.54(0.357,0.839)$ & 0.006 & $0.52(0.326,0.822)$ & $0.005 *$ \\
\hline & Secondary & $0.83(0.555,1.233)$ & 0.351 & $0.83(0.536,1.290)$ & 0.410 \\
\hline & Tertiary & $0.71(0.416,1.206)$ & 0.204 & $0.87(0.500,1.530)$ & 0.639 \\
\hline \multirow[t]{4}{*}{ Marital Status } & Never married & 1 & & 1 & \\
\hline & Married(Inc. de facto) & $1.32(0.819,2.130)$ & 0.254 & $1.34(0.797,2.251)$ & 0.270 \\
\hline & Separated or Divorced & $1.72(1.026,2.881)$ & 0.040 & $1.75(0.995,3.066)$ & 0.052 \\
\hline & Widow/Widower & $1.30(0.704,2.415)$ & 0.399 & $1.24(0.628,2.450)$ & 0.536 \\
\hline \multirow[t]{4}{*}{ Weight at ART Start } & $<=45$ & 1 & & 1 & \\
\hline & $46-51$ & $0.71(0.452,1.120)$ & 0.141 & $0.77(0.472,1.260)$ & 0.299 \\
\hline & $52-58$ & $0.73(0.471,1.134)$ & 0.162 & $0.91(0.558,1.478)$ & 0.697 \\
\hline & $59+$ & $0.76(0.486,1.174)$ & 0.212 & $0.93(0.557,1.478)$ & 0.765 \\
\hline \multirow[t]{4}{*}{ Base WHO Stage } & I & 1 & & 1 & \\
\hline & II & $1.67(1.004,2.770)$ & 0.048 & $1.82(1.056,3.145)$ & $0.031 *$ \\
\hline & III & $1.48(0.901,2.417)$ & 0.122 & $1.46(0.830,2.559)$ & 0.189 \\
\hline & IV & $1.68(0.913,3.109)$ & 0.095 & $1.62(0.785,3.346)$ & 0.191 \\
\hline \multirow[t]{3}{*}{ Base Functional Status } & Working & 1 & & & \\
\hline & Ambulatory & $0.99(0.617,1.589)$ & 0.967 & & \\
\hline & Bedridden & $0.91(0.288,2.884)$ & 0.875 & & \\
\hline \multirow[t]{5}{*}{ Base CD4 Count } & $<=50$ & 1 & & 1 & \\
\hline & $51-100$ & $0.79(0.473,1.311)$ & 0.359 & $0.74(0.425,1.286)$ & 0.285 \\
\hline & $101-200$ & $0.62(0.389,1.000)$ & 0.050 & $0.57(0.337,0.972)$ & $0.039 *$ \\
\hline & $201-350$ & $0.83(0.520,1.332)$ & 0.443 & $0.82(0.474,1.406)$ & 0.464 \\
\hline & $350+$ & $1.17(0.474,2.871)$ & 0.738 & $1.20(0.450,3.196)$ & 0.717 \\
\hline \multirow[t]{6}{*}{ Base Regimen } & TDF-NVP & 1 & & 1 & \\
\hline & TDF-EFV & $0.72(0.448,1.155)$ & 0.172 & $0.52(0.318,0.863)$ & $0.011^{*}$ \\
\hline & ZDV-EFV & $0.91(0.502,1.654)$ & 0.760 & $0.78(0.415,1.466)$ & 0.440 \\
\hline & ZDV-NVP & $0.76(0.458,1.271)$ & 0.299 & $0.69(0.403,1.174)$ & 0.170 \\
\hline & d4T-EFV & $0.74(.169,3.254)$ & 0.692 & $0.25(0.033,1.955)$ & 0.188 \\
\hline & d4T-NVP & $0.83(0.246,2.790)$ & 0.762 & $0.79(0.229,2.759)$ & 0.719 \\
\hline \multirow[t]{2}{*}{$\mathrm{CPT}$} & Yes & $0.87(0.503,1.505)$ & 0.619 & & \\
\hline & No & 1 & & & \\
\hline \multirow[t]{2}{*}{ IPT } & Yes & $0.48(0.067,3.413)$ & 0.461 & & \\
\hline & No & 1 & & & \\
\hline \multirow[t]{2}{*}{ TB Treatment } & Yes & $1.72(1.179,2.499)$ & 0.005 & $1.67(1.043,2.677)$ & $0.033^{*}$ \\
\hline & No & 1 & & 1 & \\
\hline
\end{tabular}

DOI: http://dx.doi.org/10.4314/ejhs.v27i1.5S 
Table 5: Predictors of Severe Adverse drug reactions within 6 months of ART initiation in HIV patients at tertiary hospitals in Ethiopia: ART experienced group.

\begin{tabular}{|c|c|c|c|c|c|}
\hline \multirow[t]{2}{*}{ Characteristics } & & \multicolumn{2}{|c|}{ Uni-variable analysis } & \multicolumn{2}{|c|}{ Multivariable analysis } \\
\hline & & IRR $(95 \%$ CI) & P Value & AIRR (95\% CI) & P Value \\
\hline \multirow[t]{2}{*}{ Sex } & Female & 1 & \multirow{2}{*}{\multicolumn{3}{|c|}{ NS }} \\
\hline & Male & $0.771(0.515-1.155)$ & & & \\
\hline Age ART & $18-24$ & 1 & & 1 & \\
\hline \multirow[t]{3}{*}{ Start } & $25-34$ & $1.125(0.550-2.303)$ & 0.747 & $1.142(0.556-2.345)$ & 0.717 \\
\hline & $35-44$ & $1.011(0.475-2.154)$ & 0.977 & $0.977(0.453-2.105)$ & 0.953 \\
\hline & $45+$ & $2.147(1.013-4.550)$ & 0.046 & $2.099(0.971-4.539)$ & 0.059 \\
\hline ART Start & $2005-2008$ & 1 & & 1 & \\
\hline \multirow[t]{2}{*}{ Year } & $2009-2010$ & $0.663(0.429-1.026)$ & 0.065 & $0.785(0.454-1.356)$ & 0.385 \\
\hline & 2011-2013 & $0.661(0.393-1.113)$ & 0.120 & $0.829(0.422-1.631)$ & 0.588 \\
\hline \multirow{4}{*}{$\begin{array}{l}\text { Educational } \\
\text { status }\end{array}$} & No education & 1 & & 1 & \\
\hline & Primary & $0.526(0.316-0.873)$ & 0.013 & $0.601(0.358-1.010)$ & 0.054 \\
\hline & Secondary & $0.714(0.445-1.146)$ & 0.164 & $0.901(0.549-1.478)$ & 0.681 \\
\hline & Tertiary & $0.739(0.386-1.417)$ & 0.363 & $0.975(0.501-1.897)$ & 0.941 \\
\hline Marital & Never married & 1 & & 1 & \\
\hline \multirow[t]{4}{*}{ Status } & Married(Inc. de facto) & $1.057(0.584-1.913)$ & 0.855 & $1.073(0.587-1.962)$ & 0.818 \\
\hline & Separated/Divorced & $2.107(1.157-3.838)$ & 0.015 & $2.110(1.141-3.901)$ & $0.017^{*}$ \\
\hline & Widow/Widower & $1.263(0.630-2.531)$ & 0.511 & $1.217(0.591-2.503)$ & 0.595 \\
\hline & $<=45$ & 1 & & & \\
\hline Weight at & $46-51$ & $0.984(0.584-1.658)$ & 0.952 & NS & \\
\hline \multirow[t]{2}{*}{ ART Start } & $52-58$ & $0.936(0.545-1.608)$ & 0.811 & & \\
\hline & $59+$ & $1.120(0.649-1.932)$ & 0.684 & & \\
\hline Base WHO & I & 1 & & & \\
\hline \multirow[t]{3}{*}{ Stage } & II & $0.992(0.455-2.160)$ & 0.984 & NS & \\
\hline & III & $1.174(0.600-2.297)$ & 0.639 & & \\
\hline & IV & $1.432(0.673-3.048)$ & 0.351 & & \\
\hline Base & Working & 1 & & & \\
\hline Functional & Ambulatory & $1.131(0.707-1.809)$ & 0.607 & NS & \\
\hline Status & Bedridden & $0.762(0.240-2.418)$ & 0.645 & & \\
\hline Base CD4 & $<=50$ & 1 & & & \\
\hline \multirow[t]{4}{*}{ Count } & $51-100$ & $0.866(0.445-1.685)$ & 0.672 & & \\
\hline & $101-200$ & $0.898(0.501-1.611)$ & 0.718 & & \\
\hline & $201-350$ & $1.290(0.712-2.337)$ & 0.402 & & \\
\hline & $350+$ & $0.472(0.062-3.559)$ & 0.466 & & \\
\hline Base & TDF-NVP & 1 & & 1 & \\
\hline \multirow[t]{5}{*}{ Regimen } & TDF-EFV & $0.769(0.336-1.759)$ & 0.534 & $0.544(0.232-1.277)$ & 0.162 \\
\hline & ZDV-EFV & $0.493(0.190-1.281)$ & 0.147 & $0.285(0.104-0.779)$ & $0.014 *$ \\
\hline & ZDV-NVP & $0.741(0.340-1.615)$ & 0.450 & $0.622(0.277-1.396)$ & 0.249 \\
\hline & d4T-EFV & $1.057(0.428-2.610)$ & 0.905 & $0.537(0.196-1.473)$ & 0.227 \\
\hline & d4T-NVP & $1.217(0.557-2.656)$ & 0.623 & $0.817(0.335-1.988)$ & 0.655 \\
\hline \multirow[t]{2}{*}{ CPT } & Yes & $1.350(0.675-2.701)$ & 0.396 & & \\
\hline & No & 1 & & & \\
\hline \multirow[t]{2}{*}{ IPT } & Yes & $1.619(0.705-3.719)$ & 0.256 & & \\
\hline & No & 1 & & & \\
\hline TB & Yes & $2.409(1.630-3.559)$ & 0.000 & $2.817(1.847-4.298)$ & $<0.0001 *$ \\
\hline Treatment & No & 1 & & 1 & \\
\hline
\end{tabular}

*- Statistically significant

It is thus evident that anti-tuberculosis treatment in the first 6 months of HAART in initiation was found to be the strongest independent predictor of
ADR; any form of ADR increased by $67 \%$ in those taking TB treatment (Table 3) while severe forms were found to be 3 times higher.

DOI: http://dx.doi.org/10.4314/ejhs.v27i1.5S 


\section{DISCUSSION}

Toxicities related to ART drug were widely prevalent among HIV patients on follow-up in tertiary hospitals in Ethiopia; significant proportion of them resulted in serious morbidities. Toxicities were found to be highest in the first 6 months of ART initiation. Concomitant antituberculosis treatment was found to be an independent predictor of adverse drug reaction due to ART.

All of the participants were started on combination of two NRTIs and NNRTI adhering to WHO's recommendation for resource limited settings (20). Most of patients taking $d 4 t$ were shifted to TDF since 2009 after revision of national guidelines in 2008 (16). As a result, the proportion of patients starting on $\mathrm{d} 4 \mathrm{t}$ containing regimen dropped from $70 \%$ in 2006 to $1 \%$ in 2013. However, the fact that some patients were still started on stavudin containing regimen (42 patients in 2010, 4 in 2011, 3 in 2012 and 2 in 2013) years after revision of the guideline should not pass unnoticed.

The overall prevalence of ADR of $22.1 \%$ in our study is comparable with findings from the developing world; $19.5 \%$ in Cameroon (21), $26.8 \%$ in India (22). However, the incidence rate is much higher than findings in Nigeria of 4.6 per 100 person years (23). Because of the very nature of occurrences of ADR due to ART, neither assessment of the total prevalence of the problem nor the overall incidence rate tell the exact magnitude of the problem. Longer exposure to the drugs may inflate the prevalence and overall incidence rate of ADR. However, more serious and common toxicities occur during the initial phase of ART (7). Hence, comparison between studies may not be straight forward.

Consistent with global data, incident toxicities in our study were found to be more common in the first 6 months of ART initiation (4, 7, 24). Patients with advanced disease at ART initiation in particular are at higher risk of ADR $(25,26)$. However, neither WHO stage of the disease nor $\mathrm{CD}_{4}$ count at ART initiation was associated with occurrences of ADR in our study. This may be partly explained by the fact that most the study participants had advanced WHO stage and low $\mathrm{CD}_{4}$ count at HAART initiation. Additionally, failure to detect and report milder forms of ADR may explain the absence of this relationship. Despite using standardized reporting format for any ADR, it is well documented that both highly trained professionals and patient underreport milder forms $(27,28)$. We thus believe that milder dermatologic manifestations and gastrointestinal symptoms might have been underreported by patients and overlooked by treating physician as non-life threatening event.

Early toxicities are also known to be worsened with concomitant OI treatment (29). Though INH and Cotrimoxazole prophylaxis for OIs were not associated with increased toxicities in our study, concomitant anti-tuberculosis admiration was found to be the strongest predictor of ADR. Overlapping toxicities between HAART and anti-TB drugs has been well documented and know to hamper success of the treatment (30). While this has been an issue, the benefit of addressing the two infections should take priority.

When years of ART initiation were compared, incident toxicity was found to be more common in those who started ART since 2009. This difference is mainly due to design effect. Data for patients who started ART before 2009 was obtained only retrospectively and incident toxicities could have been under reported. When only prospectively cohorts were analyzed separately, the effect of year of ART initiation disappears. We believe that the lowest incidence of toxicities in prior years was mainly due to underreporting than other reasons. Besides, as experience in HIV care has improved over the last many years in the country, detection rate of all forms of adverse reaction has expectedly improved resulting in the increment in the rate of reported ADR.

Even though this study is multicenter large scale cohort in Ethiopia, there are certain limitations worth mentioning. First of all, the data prior to 2009 were collected from medical records only. As a result, certain ADR, the non-life threatening ones, could have been underreported. Secondly, some of the adverse reactions diagnosed on clinical ground only were based on treating physician's diagnosis which might have also resulted inconsistence across sites and underreporting. Last but most importantly, the study

DOI: http://dx.doi.org/10.4314/ejhs.v27i1.5S 
was based in tertiary/university hospitals in the country with better laboratory facilities and more qualified professionals in HIV care. Hence, the findings may not be generalizable to all HIV patients in Ethiopia.

In conclusion, ADR due to ART drugs has been found to be highly prevalent in HIV patients on follow-up at tertiary hospitals in Ethiopia. A significant proportion was found to be serious adverse reactions resulting in admissions and/or regimen changes. ADRs were prominent during the first 6 months after ART initiation. Concomitant administration of full antituberculosis regimen was the strongest independent predictor for the occurrence of these toxicities. Thus, routine clinical and laboratory monitoring of patients on ART should be strengthened with particular emphasis in the first 6 months. High risk patients (those with comorbidities, advanced immunosuppression and concomitant OIs) should be actively identified before ART initiation. These patients should be effectively treated for the OIs or comorbidities, if possible, before ART initiation. Selection of ART regimen should also put into consideration their other medical conditions. Strategies should also be devised to replace older and more toxic agents with newer and safer drugs available. All facilities rendering ART service should follow standard reporting method for incident ADR related to ART and OI treatment.

\section{ACKNOWLEDGMENTS}

We are indebted to Johns Hopkins University, USA and United States Centers for Disease Control and Prevention (CDC) for funding this research and following its progress throughout. We also would like to thank the seven hospitals and their staffs for their support during the data collection. We also acknowledge EPHI for its leading role during manuscript preparation.

We would also like to express our acclaim to $\mathrm{Dr}$ Alula M. Teklu for his perseverance in taking this process to the level of publication.

\section{REFERENCES}

1. Dixon S, McDonald S, Roberts J. The impact of HIV and AIDS on Africa's economic development. Bmj. 2002;324(7331):232-4.

2. Mwagomba B, Zachariah R, Massaquoi M, Misindi D, Manzi M, Mandere BC, et al. Mortality reduction associated with HIV/AIDS care and antiretroviral treatment in rural Malawi: evidence from registers, coffin sales and funerals. PloS one. 2010;5(5):e10452.

3. Pau AK. Antiretroviral Therapy-associated Serious and Life-threatening Toxicities. Current infectious disease reports. 2003;5(5):429-38.

4. Masenyetse LJ, Manda SO, Mwambi HG. An assessment of adverse drug reactions among HIV positive patients receiving antiretroviral treatment in South Africa. AIDS research and therapy. 2015;12:6.

5. International drug monitoring: the role of national centres. Report of a WHO meeting. World Health Organization technical report series. 1972;498:1-25.

6. Murphy RA, Sunpath H, Kuritzkes DR, Venter F, Gandhi RT. Antiretroviral therapyassociated toxicities in the resource-poor world: the challenge of a limited formulary. The Journal of infectious diseases. 2007;196 Suppl 3:S449-56.

7. Subbaraman R, Chaguturu SK, Mayer KH, Flanigan TP, Kumarasamy N. Adverse effects of highly active antiretroviral therapy in developing countries. Clinical infectious diseases : an official publication of the Infectious Diseases Society of America. 2007;45(8):1093-101.

8. O'Brien ME, Clark RA, Besch CL, Myers L, Kissinger P. Patterns and correlates of discontinuation of the initial HAART regimen in an urban outpatient cohort. Journal of acquired immune deficiency syndromes. 2003;34(4):407-14.

9. Wube M, Tesfaye A, Hawaze S. Antiretroviral Therapy Regimen Change Among HIV/AIDS Patients in Nekemt Hospital: a Primary Care Hospital in Oromia Regional State, Ethiopia. .

DOI: http://dx.doi.org/10.4314/ejhs.v27i1.5S 
Journal of Applied Pharmaceutical Science 2013;3(8):5.

10. Njuguna C, Orrell C, Kaplan R, Bekker LG, Wood R, Lawn SD. Rates of switching antiretroviral drugs in a primary care service in South Africa before and after introduction of tenofovir. PloS one. 2013;8(5):e63596.

11. Hartmann M. The side effects of antiretroviral therapy. Hautarzt. 2006;57(11):6.

12. Yunihastuti E, Widhani A, Karjadi TH. Drug hypersensitivity in human immunodeficiency virus-infected patient: challenging diagnosis and management. Asia Pacific allergy. 2014;4(1):54-67.

13. Shet A, Antony J, Arumugam K, Kumar Dodderi S, Rodrigues R, DeCosta A. Influence of adverse drug reactions on treatment success: prospective cohort analysis of HIV-infected individuals initiating first-line antiretroviral therapy in India. PloS one. 2014;9(3):e91028.

14. Mehta U, Durrheim DN, Blockman M, Kredo T, Gounden R, Barnes KI. Adverse drug reactions in adult medical inpatients in a South African hospital serving a community with a high HIV/AIDS prevalence: prospective observational study. British journal of clinical pharmacology. 2008;65(3):396-406.

15. UNAIDS. AIDS epidemic update: the latest epidemiology data. 2014 [cited 201430 August]. Available from: www.unaids.org

16. Federal ministry of health. Guideline for the management of opportunistic infections and antiretroviral treatment in adolescents and adults in Ethiopia. Federal HIV/AIDS Prevention and Control Office Federal Ministry of Health. Addis Ababa, Ethiopia2008.

17. Global fund. Global fund mid-2014 results 2014 [cited 201430 August]. Available from: http://www.theglobalfund.org/documents/resu 1ts/Results_2014-07-17_Factsheet_en/

18. Abdissa SG, Fekade D, Feleke Y, Seboxa T, Diro E. Adverse drug reactions associated with antiretroviral treatment among adult Ethiopian patients in a tertiary hospital. Ethiopian medical journal. 2012;50(2):107-13.

19. Jima $Y$, Angamo $M$, Wabe $N$. Causes for antiretroviral regimen change among HIV/AIDS patients in Addis Ababa, Ethiopia.
Tanzania Journal of Health Research 2013;15(1).

20. Consolidated Guidelines on the Use of Antiretroviral Drugs for Treating and Preventing HIV Infection: Recommendations for a Public Health Approach. WHO Guidelines Approved by the Guidelines Review Committee. Geneva2013.

21. Namme Luma H, Doualla MS, Choukem SP, Temfack E, Ashuntantang G, Achu Joko H, et al. Adverse drug reactions of Highly Active Antiretroviral Therapy (HAART) in HIV infected patients at the General Hospital, Douala, Cameroon: a cross sectional study. The Pan African medical journal. 2012;12:87.

22. Divakar B, Mistry S, Kantharia N, Mamtarani. The Study of Adverse Drug Reactions (ADR's) in HIV patients taking Highly Active Antiretroviral Therapy in ART centre, $\mathrm{NCH}$, Surat, India. . Int $\mathrm{J}$ Med Pharm Sci 2012;3(2):10.

23. Eluwa GI, Badru T, Agu KA, Akpoigbe KJ, Chabikuli O, Hamelmann C. Adverse drug reactions to antiretroviral therapy (ARVs): incidence, type and risk factors in Nigeria. BMC clinical pharmacology. 2012;12:7.

24. Bezabhe WM, Bereznicki LR, Chalmers L, Gee P, Kassie DM, Bimirew MA, et al. Adverse Drug Reactions and Clinical Outcomes in Patients Initiated on Antiretroviral Therapy: A Prospective Cohort Study From Ethiopia. Drug safety. 2015;38(7):629-39.

25. Lichtenstein KA, Armon C, Buchacz K, Chmiel JS, Moorman AC, Wood KC, et al. Initiation of antiretroviral therapy at CD4 cell counts $>/=350$ cells $/ \mathrm{mm} 3$ does not increase incidence or risk of peripheral neuropathy, anemia, or renal insufficiency. Journal of acquired immune deficiency syndromes. 2008;47(1):27-35.

26. Prosperi MC, Fabbiani M, Fanti I, Zaccarelli M, Colafigli M, Mondi A, et al. Predictors of first-line antiretroviral therapy discontinuation due to drug-related adverse events in HIVinfected patients: a retrospective cohort study. BMC infectious diseases. 2012;12:296.

27. Hazell L, Shakir SA. Under-reporting of adverse drug reactions : a systematic review. Drug safety. 2006;29(5):385-96. 
28. Lopez-Gonzalez E, Herdeiro MT, Figueiras A. Determinants of under-reporting of adverse drug reactions: a systematic review. Drug safety. 2009;32(1):19-31.

29. Montessori V, Press N, Harris M, Akagi L, Montaner JS. Adverse effects of antiretroviral therapy for HIV infection. CMAJ : Canadian Medical Association journal $=$ journal $\mathrm{de}$
l'Association medicale canadienne. 2004;170(2):229-38.

30. Dean GL, Edwards SG, Ives NJ, Matthews G, Fox EF, Navaratne L, et al. Treatment of tuberculosis in HIV-infected persons in the era of highly active antiretroviral therapy. Aids. 2002;16(1):75-83. 